\title{
A study of genetic association with electrophysiological measures related to alcoholism: GAWI 4 data
}

\author{
Ao Yuan*1,2, Victor Apprey ${ }^{1,2}$, Jules P Harrell ${ }^{3}$, Robert E Taylor ${ }^{5}$ and \\ George E Bonney ${ }^{1,2,4}$
}

\begin{abstract}
Address: ${ }^{1}$ National Human Genome Center, Howard University, Washington DC, USA, 2 Department of Community Health and Family Medicine, Howard University, Washington DC, USA, ${ }^{3}$ Department of Psychology, Howard University, Washington DC, USA, ${ }^{4}$ Division of Medical Genetics, Department of Pediatrics, Howard University, Washington DC, USA and ${ }^{5}$ Alcoholism Research Center, Howard University, Washington DC, USA

Email: Ao Yuan* - ayuan@howard.edu; Victor Apprey - vapprey@ howard.edu; Jules P Harrell - jharrell@howard.edu; Robert E Taylor -rtaylor@howard.edu; George E Bonney -ge_bonney@howard.edu

* Corresponding author
\end{abstract}

from Genetic Analysis Workshop 14: Microsatellite and single-nucleotide polymorphism

Noordwijkerhout, The Netherlands, 7-10 September 2004

Published: 30 December 2005

BMC Genetics 2005, 6(SuppI I):SI26 doi:10.1 186/I47I-2I56-6-SI-SI26

\begin{abstract}
Recently, alcohol-related traits have been shown to have a genetic component. Here, we study the association of specific genetic measures in one of the three sets of electrophysiological measures in families with alcoholism distributed as part of the Genetic Analysis Workshop I4 data, the NTTH (non-target case of Visual Oddball experiment for 4 electrode placements) phenotypes: ntthI, ntth2, ntth3, and ntth4. We focused on the analysis of the 786 Affymetrix markers on chromosome 4. Our desire was to find at least a partial answer to the question of whether ntth I, ntth2, ntth3, and ntth4 are separately or jointly genetically controlled, so we studied the principal components that explain most of the covariation of the four quantitative traits. The first principal component, which explains $70 \%$ of the covariation, showed association but not genetic linkage to two markers: tsc0272102 and tsc0560854. On the other hand, ntthl appeared to be the trait driving the variation in the second principal component, which showed association and genetic linkage at markers in four regions: tsc0045058, tscl2 I338I, tsc0055068, and tsc005I777 at map distances 53.26, 85.42, 89.31, and 172.86, respectively. These results show that the partial answer to our starting question for this brief analysis is that the NTTH phenotypes are not jointly genetically controlled. The component ntth I displays marked genetic linkage.
\end{abstract}

\section{Background}

Recently, evidence has been found to relate alcoholism to genetic factors [1-4]. The Collaborative Study on the Genetics of Alcoholism (COGA) is a program to study this phenomenon extensively. For the Genetic Analysis Workshop 14 (GAW14), an expanded dataset was released for analysis. It contains multiple phenotypes and genomewide scans. We chose to study genetic association of electrophysiological measures related to alcoholism focusing on the NTTH phenotypes and the 786 Affymetrix single- nucleotide polymorphisms (SNPs) on chromosome 4. This chromosome has been shown to be involved in NTTH phenotypes in some previous studies.

There are four NTTH phenotypes: ntth1, ntth2, ntth3, and ntth4. For the four correlated traits a natural question is, are they mediated by the same set of genes, or, is each separately genetically controlled? Here we attempt to find a partial answer to these questions. 
Table I: Factor loadings for first two principal components

\begin{tabular}{lllll}
\hline & ntthl & ntth2 & ntth3 & ntth4 \\
\hline I st component $^{\text {nd }} 0.338$ & 0.515 & 0.569 & 0.545 \\
& 0.930 & 0.294 & 0.200 & 0.000 \\
\hline
\end{tabular}

\section{Methods}

In view of our desire to determine whether ntth1, ntth2, ntth3, and ntth4 are separately or jointly genetically controlled, we shall study the principal components that explain most of the covariation of the four quantitative traits. We shall then analyze the promising components separately.

For the association and linkage analyses, we used our Genetic Epidemiology Models (GEMs) package, which has routines for the regressive models for quantitative traits $[5,6]$. Typically for this problem, one may perform a linkage analysis to pinpoint the highly spurious region, but this is computationally intensive and time consuming. In this dataset, the number of SNPs is large. For chromosome 4 alone there are 786 SNPs. We did a two-stage analysis. The first stage is an association analysis in which we regressed the trait on age, sex, and the SNPs, one at a time, across all the 786 SNPs on chromosome 4. Strong statistical association between the phenotypes and the SNPs provided us the basis to select the phenotypes/SNPs for the next stage of analysis. In the second stage, a formal linkage analysis was performed on the SNPs selected from the first stage.

\section{Results}

The proportion of the total variance explained by the first, second, third and fourth components are: 0.698, 0.195, 0.084 , and 0.024 , respectively.

The factor loadings for the first and second principal components are listed in Table 1. The first loading shows a rather even spread, and along with the amount of covariance explained by principal components, explains almost $70 \%$ of the covariation among the four traits. The loadings for the second component, which explains only
$19.5 \%$ of the covariation, are quite illuminating. The relatively huge loading of 0.930 for ntth 1 suggests that nth 1 itself rather than the combination with the others is the important trait. So we first analyzed the first principal component, which explains almost $70 \%$ of the covariation, i.e.,

\subsection{8 ntth $1+0.515$ ntth $2+0.569$ ntth $3+0.545$ ntth 4 .}

Instead of analyzing the second principal component, which, explains $20 \%$ of the covariation, we analyzed ntth1, which is the driving factor. The third and fourth principal components account for little covariation so they were not analyzed. However, we also performed univariate linkage analysis on ntth2, ntth3, and ntth4 for comparison.

The results of the association study for the first principal component are summarized in Figure 1A, which displays the values of chi-square ratios for association versus no association for each SNP. The four horizontal lines indicate the significant levels. We find that at the 0.0001 level there are five SNPs showing significant associations. These five sit roughly around locations 60, 70, 450, 470, and 690.

The association analysis results for ntth1 alone are displayed in Figure 1B. We see ten SNPs significant at the 108 level. This is a far stronger result than those using the principal component combinations. Although Figures $1 \mathrm{~A}$ and $1 \mathrm{~B}$ show many peaks, the remarkable difference is that the markers around position 300 are prominent in ntth1 but not in the first principal component.

Table 2 presents the linkage analysis results for those SNPs that showed significant association at the 0.00001 level in the first stage of analysis. The very low level of significance was chosen because of concerns about multiple comparison. The LOD scores and chi-square values in Table 2 are from the second stage of linkage analysis. The marker tsc0560854 at position 469 had significant chi-square value at the 5\% level, but it is not significant by the conventional linkage standard of LOD score 2.0 or higher. The results suggest that the principal components method is not adequate for this problem. It means that using the

Table 2: Linkage results on chromosome 4 for the first principal component of $n$ tth

\begin{tabular}{cccccc}
\hline SNP & Marker name & Map distance & Allele & LOD Score & Chi-square value \\
\hline 60 & tsc0273260 & 28.64 & 1 & 0.00 & 0.00 \\
79 & tsc0272102 & 32.91 & 1 & 0.44 & 0.00 \\
451 & tsc0538170 & 120.48 & 1 & 0.85 & 0.00 \\
469 & tsc0560854 & 122.85 & 1 & 0.00 & 3.93 \\
684 & tsc0047209 & 166.94 & 1 & 0.00 \\
\hline
\end{tabular}


A) First Principal Component of ntth

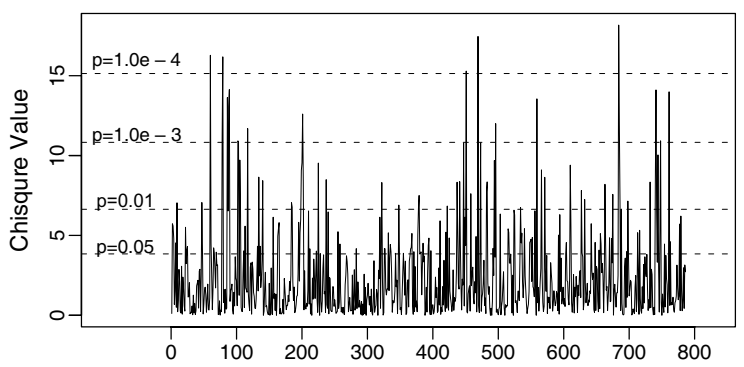

B) ntth1 Data Only

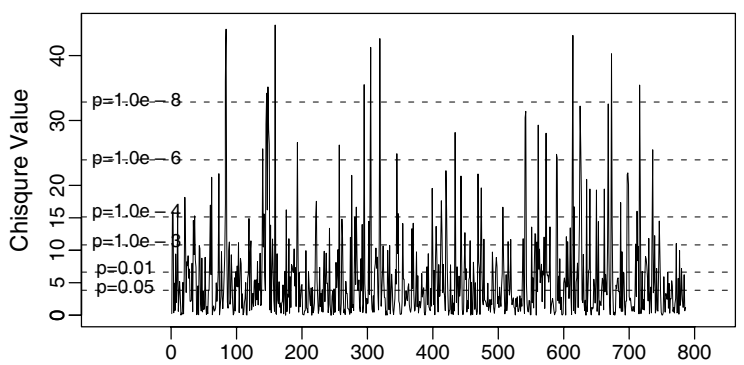

Figure I

ntth chi-square values for chromosome 4 SNPs.

first principal linear combination of the phenotypes may have smoothed/filtered out the linkage effect.

The linkage results for ntth 1 alone are presented in Table 3. It is interesting to note that the marker tsc0560854 at position 469 , which showed significant chi-square value in the first principal component, were not significant for ntth1 and so are not in Table 3. On the other hand, markers tsc0045058 at position 148, tsc1213381 at position
295 , tsc0055068 at position 319 , and tsc0051777 at position 714 showed significant linkage; these markers were at map distances 53.26, 85.42, 89.31, and 172.86, respectively. For ntth1, other significant linkage LOD score peaks occur at tsc1276837 (map distance 34.24), tsc0526379 (map distance 52.99), and at tsc0527513 (map distance 55.57). Also, there are some SNPs showing significant association in Figure 1B, but not all of them showing evidence of linkage as in Table 3 . We think the significant association in the first stage of analysis indicate some non-random association between the phenotype and the SNPs, which may suggest Hardy-Weinberg disequilibrium among the SNPs or somthing else, not necessarily linkage.

The linkage analysis results for ntth2, ntth3, and ntth 4 are presented in Table 4, showing no linkage to the selected markers that showed significant association with the trait in the first stage of analysis.

\section{Conclusion}

Our analysis of the four NTTH phenotypes, although brief, is revealing. The first principal component, which explains $70 \%$ of the covariation, showed association but not linkage to two markers: tsc0272102 and tsc0560854. On the other hand, ntth1, which was the trait driving the variation in the second principal component, showed association and linkage at markers in four regions: tsc0045058, tsc1213381, tsc0055068, and tsc0051777 at map distances 53.26, 85.42, 89.31, and 172.86 respectively.

\section{Abbreviations}

COGA: Collaborative Study on the Genetics of Alcoholism

GAW14: Genetic Analysis Workshop 14

SNP: Single-nucleotide polymorphism

Table 3: Linkage results on chromosome 4 for ntth I

\begin{tabular}{|c|c|c|c|c|c|}
\hline SNP & Marker name & Map distance & Allele & LOD score & Chi-square value \\
\hline 84 & tsc 1276837 & 34.24 & 2 & 1.75 & 8.05 \\
\hline 146 & tsc0526379 & 52.99 & 1 & 1.92 & 8.87 \\
\hline 148 & tsc0045058 & 53.26 & I & 3.35 & 15.45 \\
\hline 159 & tsc 0527513 & 55.57 & 2 & 1.93 & 8.89 \\
\hline 295 & tscl $21338 \mid$ & 85.42 & 2 & 2.22 & 10.25 \\
\hline 305 & tsc $07440 \mid 3$ & 87.05 & 2 & 0.75 & 3.48 \\
\hline 319 & tsc0055068 & 89.31 & 2 & 3.03 & 13.97 \\
\hline 614 & tsc004485I & 150.40 & 2 & 0.43 & 1.97 \\
\hline 673 & tsc0572265 & 165.77 & 1 & 0.00 & 0.00 \\
\hline 714 & tsc005I777 & 172.86 & 2 & 2.57 & 11.83 \\
\hline
\end{tabular}


Table 4: Linkage results on chromosome 4 for ntth2-ntth4

\begin{tabular}{rrrrrrr}
\hline & SNP & Marker name & Map distance & Allele & LOD score & Chi-square value \\
\hline \multirow{2}{*}{ ntth2 } & 88 & tsc0750487 & 34.6124 & I & 0.00 & 0.00 \\
ntth3 & 89 & tsc0568024 & 34.6135 & 1 & 0.00 & 0.00 \\
ntth4 & 732 & tsc0540150 & 177.197 & 1 & 0.00 & 0.00 \\
& 89 & tsc0568024 & 34.6135 & 1 & 0.00 & 0.21 \\
\hline
\end{tabular}

\section{Authors' contributions}

AY worked on the methodology, participated in the computations, and drafted the manuscript. VA implemented the computer program and participated in the computations. JPH participated in the analysis. RET participated in the analysis and interpretation of results. GEB helped to decide on the methodology, participated in the analysis, and assisted in writing the manuscript.

\section{Acknowledgements}

The research was supported in part by public health research grants from the National Institutes of Health (AGI6996, AA0 14643), and National Center for Research Resources (2GI2RR003048).

\section{References}

I. Long JC, Knowler WC, Hanson RL, Robin RW, Urbanek M, Moore E, Bennett PH, Golman G: Evidence for genetic linkage to alcohol dependence on chromosome 4 and II from an autosomewide scan in an American Indian population. Am J Med Genet 1998, 81:216-221.

2. Reich T, Edenberg HJ, Goate A, Williams JT, Rice JP, Van Eerdewegh P, Foroud T, Hesselbrock V, Schuckit MA, Bucholz K, Porjesz B, Li TK, Conneally PM, Nurnberger JI Jr, Tischfield JA, Crowe RR, Cloninger CR, Wu W, Shears S, Carr K, Crose C, Willig C, Begleiter H: Genome-wide search for genes affecting the risk for alcohol dependence. Am J Med Genet 1998, 81:207-2I5.

3. Zinn-Justin A, Abel L: Genome search for alcohol dependence using the weighted pair wise correlation linkage method: interesting findings on chromosome 4. Genet Epidemiol 1999, I7(SuppI I):S42I-S426.

4. Windemuth C, Hahn A, Strauch K, Bauer MP, Wienker T: Linkage analysis in alcohol dependence. Genet Epidemiol 1999, I 7(Suppl I):S403-S407.

5. Bonney GE: On the statistical determination of major gene mechanisms in continuous human traits: regressive models. Am J Med Genet 1984, 18:731-749.

6. Bonney GE, Lathrop M, Lalouel J-M: Combined linkage and segregation analysis using regressive models. Am J Hum Genet 1988, 43:29-37.

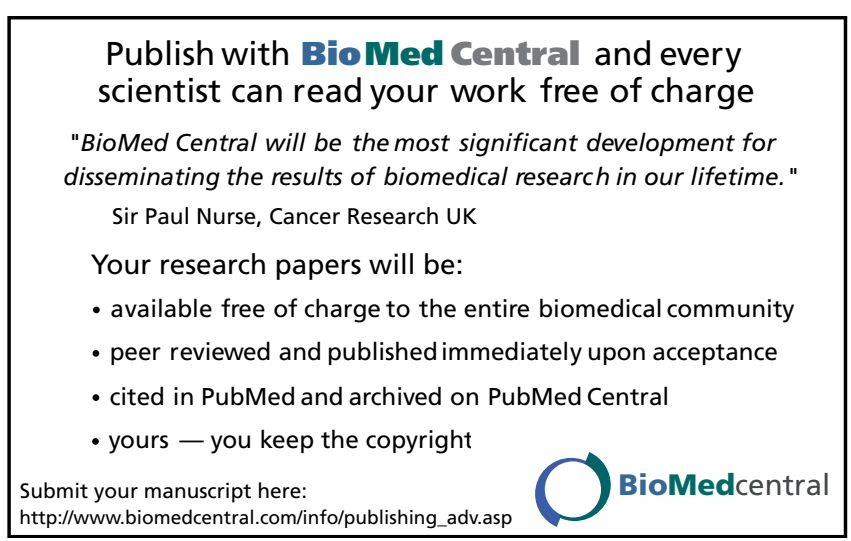

